\title{
Propuesta de reconstrucción 3d de objetos basado en la selección de los mejores parámetros de la Wavelet Morlet
}

\author{
Claudia Victoria López ${ }^{1}$, María Eugenia Quintanar Pérez ${ }^{3}$, \\ |Jesús Carlos Pedraza Ortega ${ }^{2}$, Gonzalo Elías Blanco Silva ${ }^{1}$, \\ Juan Manuel Ramos Arreguín ${ }^{2}$, Efrén Gorrostieta Hurtado² \\ ${ }^{1}$ Facultad de Informática, Universidad Autónoma de Querétaro, Querétaro, México \\ ${ }^{2}$ Facultad de Ingeniería, Universidad Autónoma de Querétaro, Querétaro, México \\ ${ }^{3}$ Instituto Tecnológico de Querétaro, Querétaro, México
}

azul.cielo.2007@gmail.com,mequintanar@hotmail.com, caryoko@yahoo.com, gblanco25@alumnos.uaq.mx,jramos@mecamex.net, efrengorrostieta@gmail.com

\begin{abstract}
Resumen. Entre los métodos de proyección de luz estructurada para el proceso de reconstrucción 3D, se encuentran el de Perfilometría de Fourier y Perfilometría Wavelet, siendo el primero el más utilizado. Este trabajo muestra una comparativa entre el desempeño de la Perfilometría Wavelet y la de Fourier, implementando la selección de los mejores parámetros para la wavelet Morlet, Considerando el análisis de la estimación de fase mediante la frecuencia espacial fo, la cual es establecida por el número de franjas y sirve como parámetro para llevar a cabo el filtrado en esa frecuencia dentro de la Wavelet Morlet. La metodología aplicada utiliza la selección de objetos reales y virtuales, así como la determinación de la frecuencia espacial en caso de no tenerla como parámetro de entrada. Se llevaron a cabo pruebas primero con objetos virtuales y se obtuvieron los parámetros en donde el error en la reconstrucción 3D es menor. Los resultados obtenidos con estos parámetros fueron implementados para la reconstrucción $3 \mathrm{D}$ en objetos reales.
\end{abstract}

Palabras clave: reconstrucción 3D, wavelet Morlet, parámetros, perfilometria de Fourier.

\section{D Object Reconstruction Proposal Based on the Morlet Wavelet best parameter selection}

\begin{abstract}
Among the structured light projection methods for the 3D reconstruction process, there are the Fourier and Wavelet based Profilometry, being Fourier the most used. In this work we present a comparative between Fourier and Wavelet Profilometry performance by means of the selection of the best parameters of the Morlet Wavelet. Analyzing the phase estimation by using the fo spatial frequency, which is established by the fringe numbers and serves as
\end{abstract}




\begin{abstract}
a parameter to perform the frequency filtering with the Morlet Wavelet. The proposed methodology is applied and it uses the selection of virtual and real objects, later, the spatial frequency is determined and in case of not having this frequency it is obtained and used later in the methodology. Some test were carried out firstly with virtual objects, here the parameters that lead to the minimum error in the $3 \mathrm{D}$ reconstruction are obtained. The results with the obtained parameters were implemented in the $3 \mathrm{D}$ reconstruction of real objects.
\end{abstract}

Keywords: 3D reconstruction, Morlet wavelet, parameter selection, Fourier profilometry.

\title{
1. Introducción
}

El avance tecnológico se ha extendido, abarcando áreas significativas que anteriormente no se habían considerado, debido a la falta del desarrollo de herramientas necesarias para el estudio de estos campos. Este avance ha generado demandas de nuevos métodos para mejorar la calidad de los procesos, los cuales, son cada vez más sofisticados y precisos, haciendo uso de herramientas que implementan una mejora continua. Dentro de este desarrollo, el uso de las técnicas de procesamiento digital de imágenes se ha incrementado en las últimas décadas, mostrando progresos significativos en el área de reconstrucción de objetos 3D. Existen dos clasificaciones dentro de la medición de objetos las cuales son técnicas de contacto o no contacto. Las técnicas de no contacto son las más utilizadas por sus características no invasivas y su velocidad de procesamiento. De esta técnica deriva el método de luz estructurada, del cual parte el de proyección de franjas, basado en la proyección de franjas emitido por un proyector sobre un objeto, donde posteriormente, el efecto de la distorsión de dichas franjas es capturado por una cámara. A. Asundi, Z. Wensen, 1998 [1].

Entre la variedad de métodos que consideran la proyección de patrones espaciales sinusoidales, existen técnicas que ofrecen mejores resultados en la extracción de distribuciones de fase de dichos patrones bi-dimensionales deformados: uno de ellos es llamado cambio de fase (phase-shifting) y el otro Perfilometría de Fourier (Fourier Transform Profilometry o FTP), éste último propuesto originalmente por Takeda [2] y posteriormente modificado y mejorado por $\mathrm{Wu} \mathrm{Lu} \mathrm{[3]} \mathrm{y} \mathrm{Pedraza} \mathrm{[4].} \mathrm{La} \mathrm{diferencia} \mathrm{entre}$ estos métodos es que el primero requiere de al menos 3 imágenes para obtener la información de fase del objeto. Por otro lado, la FTP extrae la información del mapa de profundidad (el cual contiene la información de la altura del objeto) utilizando una sola imagen, lo que lo ha convertido en un método popular para el análisis de patrones de franjas. Sin embargo, independientemente de las modificaciones implementadas en este método, una de las desventajas que muestra es su localización espacial pobre. El uso del primer armónico es afectado por el ruido local y la discontinuidad de los puntos, los cuales al final arrojaran un error en la fase, este error se incrementa cuando este primer armónico es superpuesto por otros altos armónicos, imposibilitando la extracción del primero. Zhang [5], Con estas altas frecuencias existe un traslape en la etapa del desdoblamiento de fase, generando que la altura estimada del objeto se extienda de forma global, obteniendo una reconstrucción 3D de objetos no adecuada. [4, 6-8]. 
Debido a que la FTP trabaja solo con señales estacionarias existe una pérdida de información en la localización en el tiempo de las frecuencias de los componentes dados.

Otra técnica que obtiene el mapa de profundidad partiendo de una sola imagen es la Perfilometría Wavelet (WTP), la cual trabaja con Transformada Wavelet, sobresaliendo por su capacidad de procesamiento de señales no estacionarias, además de la localización de dominio de tiempo frecuencia. Este concepto fue introducido por Zhong et al en 2004 [9]. La extracción de fase de la WTP maneja dos enfoques, uno es el método de fase gradiente y el segundo es la estimación de fase. Este trabajo utiliza éste último debido a que este método supera al de gradiente de fase para un patrón con o sin ruido. Gdeisat [10].

El uso de la WTP está siendo muy investigado, con un gran número de trabajos que le preceden, Como es el caso de Xiang [11] y Qiang Zhang [12], donde ambos proponen el uso de las Wavelets Haar y Morlet para obtener la información de altura de los objetos. Abdulbasit Z, et al en 2008 [13] propone la transformada continua Morlet modificada en 1D para el análisis de patrón de franjas. Sergio F, et al, 2011 [14], utiliza el análisis de patrón de franjas haciendo una comparación de las técnicas principales de tiempo-frecuencia (Transformada de Fourier, ventaneo de Fourier y Transformada Wavelet: Shannon, B-Spline, Paul y Morlet), trabajando con objetos reales y virtuales mostrando resultados cualitativos y cuantitativos. Por ello, este trabajo propone el uso de la Perfilometria Wavelet haciendo una comparación con la Perfilometria de Fourier. La transformada Morlet es usada con parámetros específicos con la finalidad de obtener una mejor reconstrucción, tomando como ventaja que la transformada wavelet trabaja con señales no estacionarias, así como su análisis multiresolución; de esta forma el proceso de reconstrucción 3D es llevado a cabo con ambos métodos en 1D, para verificar cuál de los dos presenta un mejor desempeño en la reconstrucción de objetos virtuales, y así, reproducir ambos resultados en objetos reales.

\section{Perfilometría de Fourier y Wavelet}

El método de Perfilometría de Fourier (FTP) es uno de los más importantes dentro del conjunto de técnicas que existen para la adquisición de imágenes 3D. La idea básica del método consiste en proyectar un patrón de franjas sobre el objeto del que se quiere obtener su imagen, al grabar con una cámara la imagen de la escena obtenemos un patrón distorsionado que lleva consigo toda la información 3D del objeto particular.

Aplicando la transformada de Fourier al objeto con el patrón de franjas distorsionado, se obtiene un espectro de amplitud y uno de fase. Posteriormente se lleva a cabo un filtrado en la frecuencia espacial $f_{0}$ donde esta frecuencia corresponde al número de franjas proyectado sobre el objeto. Aplicando este filtro a ambos espectros y llevando a cabo una traslación de la imagen filtrada hacía el centro de la imagen, se obtiene la transformada inversa de Fourier. Entonces se aplica la transformada inversa de Fourier y se lleva a cabo el proceso de desdoblamiento de fase a la parte imaginaria para poder obtener así la forma o altura del objeto. Este método ha sido investigado ampliamente desde mediados de 1980, aunque tiene múltiples desventajas, ya que en ocasiones no es posible obtener buenos resultados debido a que no se obtiene de forma 
apropiada la frecuencia $f_{0}$ o bien el filtro en esa frecuencia no es muy preciso y por el traslape de frecuencias con armónicos superiores o con la componente de DC, lo cual acarrea valores no deseados en la etapa de desdoblamiento de fase. Por último, en cuando a los algoritmos de desdoblamiento de fase, también son una limitante ya que se requiere que el mapa de fase tenga el menor número de transiciones de alta frecuencias para que no afecte al momento de obtener la forma o altura del objeto.

La wavelet es una herramienta utilizada para el análisis de señales no estacionarias y variantes en el tiempo. La forma de onda de las wavelets es lo que le permite no limitarse a las funciones seno y coseno. Por otro lado tienen la capacidad de multiresolución, trasladando la señal sobre el eje x y alargando o contrayendo dicha señal en el eje y, llamando a este evento escalamiento de la señal, Cortes et al, 2007 [12]. De forma general, la Transformada Wavelet WT de una señal $\mathrm{f}$ es un conjunto de coeficientes W(a, b), cuyos índices se asocian con la escala y la posición de la señal. Considerando el caso unidimensional, los coeficientes se obtienen como:

$$
W_{(a, b)}=\int_{-\infty}^{\infty} f(t) \frac{1}{\sqrt{a}} \psi\left(\frac{x-b}{a}\right) d t
$$

donde, $a \in \mathrm{R}^{+}\{-0\}, \mathrm{y} b \in \mathbb{R}$.

La transformada continua wavelet CWT se considera como la suma sobre todo el intervalo de tiempo de la señal, multiplicada por las versiones de escala y traslación de la función $\psi$. Arellano [16] La señal original es obtenida cuando se multiplica cada uno de los coeficientes por la wavelet escalada y trasladada. Sin embargo, es casi imposible llevar a cabo el cálculo de todas las combinaciones posibles entre los factores de escala, coeficientes y traslaciones.

$$
\mathrm{C}(\text { escala, posición })=\int_{-\infty}^{\infty} f(t) \psi(\text { escala }, \text { posición, } t) d t
$$

Al trabajar con regiones de escala-tiempo el análisis wavelet permite el uso de intervalos largos en el tiempo brindando información precisa de baja frecuencia e información de alta frecuencia utilizando regiones pequeñas. Esta capacidad de convergencia y adaptabilidad se debe a su tendencia asimétrica e irregular, es decir, que la forma de onda de la wavelet pende de la variación de los coeficientes, determinando cómo la señal original será reconstruida.

El cambio de escala está representado por:

$$
\frac{1}{\sqrt{a}} \psi\left(\frac{x}{a}\right)
$$

donde $\mathrm{a}>0$.

Por otro lado, la translación se define por:

$$
\psi(x-b)
$$

entonces la traslación y el cambio de escala en una dimensión está definida por: 


$$
\psi_{a, b}=\frac{1}{\sqrt{a}} \psi\left(\frac{x-b}{a}\right)
$$

donde, $\mathrm{a}>0, \mathrm{~b} \in \mathbb{R}$.

Por lo tanto, el análisis continuo está dado por:

$$
C_{(a, b)}=\int_{R} f(t) \frac{1}{\sqrt{a}} \psi\left(\frac{x-b}{a}\right) d t,
$$

donde, $a \in R^{+}\{-0\}, y b \in \mathbb{R}$.

De aquí tenemos que:

$$
W_{(a, b)}=\int f(t) \psi_{a, b}^{*}(t) d t
$$

Debido a estas variaciones de coeficientes surge la familia de wavelets, entre las que se encuentran, B-Spline, Paul, Fan, Morlet, Mexican Hat, Haar, entre otras. Además de la familia de wavelets, éstas también se clasifican en complejas y no complejas, esta investigación usará la wavelet compleja, debido a que ésta trabaja con la parte real e imaginaria, mismas que se utilizan para obtener la amplitud y la información de fase del objeto a reconstruir.

\section{Metodología propuesta}

La metodología propuesta para el proceso de reconstrucción $3 \mathrm{D}$, se muestra en la Fig. 1. Haciendo uso de los métodos de Perfilometria de Fourier y Perfilometria Wavelet para realizar la comparativa de dicha reconstrucción entre ambos métodos. Aunque la literatura muestra que las reconstrucciones se validan comparando el objeto reconstruido con las mediciones del objeto real, lo que hace que este proceso pueda resultar costoso en su momento. Debido a esto se propone hacer esta comparativa con ambos métodos al mismo objeto. La metodología, la cual consta de 7 pasos, también incluye la identificación de si el objeto capturado es real o virtual, permitiendo con esto mejorar el proceso.

En el primer paso del proceso se adquiere la imagen a reconstruir En el segundo paso se determina si la imagen adquirida es virtual o real; para ello, se realiza un conteo de la cantidad de cambios de color que haya en el primer renglón de cada imagen, si hay más de dos cambios de color en ella, entonces, se concluye que la imagen es real y que tiene franjas proyectadas. En caso de que la imagen adquirida sea virtual, se le suma el patrón de distorsión de franjas seleccionado. Para ambos casos, la imagen adquirida es redimensionada a una de 512 x 512 pixeles, con la finalidad de tener consistencia durante todo el proceso. El tercer paso para el caso de la imagen real es calcular el número de franjas proyectadas sobre el objeto, debido a que existe una relación entre el número de franjas proyectadas y la frecuencia $f_{0}$.

Para el caso del objeto virtual es determinar la frecuencia $f_{0}$. La $f_{0}$ brinda la información correspondiente a la altura el objeto en cada pixel y contribuye a su 
reconstrucción 3D. Una vez identificado el tipo de objeto, el número de franjas y su frecuencia $f_{0}$. El cuarto paso es aplicar al objeto ambos métodos: Perfilometria de Fourier y Perfilometria Wavelet, para la wavelet se seleccionaron los mejores parámetros. En el quinto paso se obtiene la fase envuelta, donde se encuentra, la información de la altura del objeto. Dicha información está envuelta entre los valores de $-\pi$ a $\pi$, por lo que es necesario implementar un análisis de desdoblamiento de fase. La fase desenvuelta es obtenida en el sexto paso, es en este punto donde el análisis es aplicado. Se considera el algoritmo de desdoblamiento de fase propuesto por Itoh, 1982 [8], el cual permite obtener la profundidad del objeto.

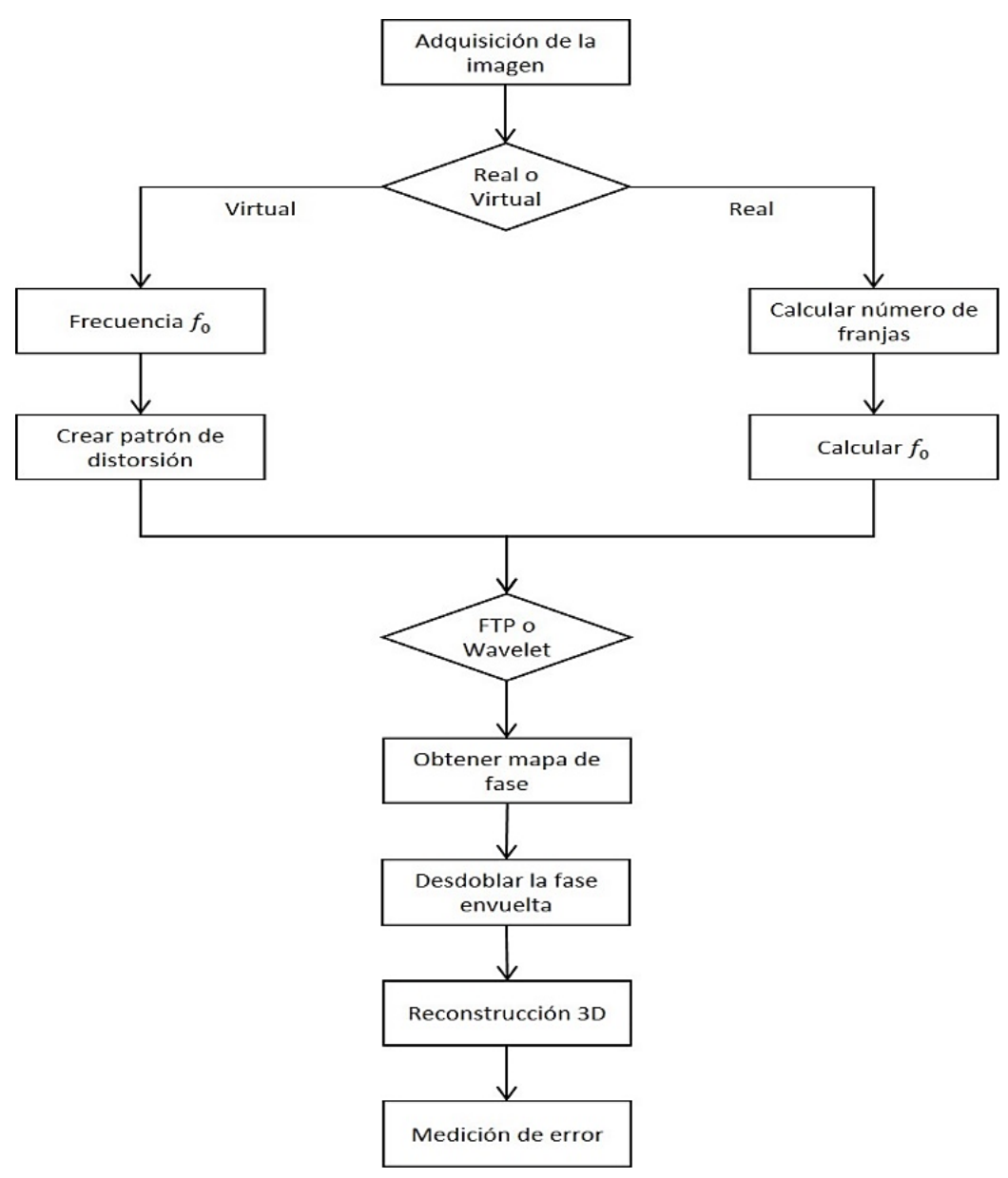

Fig. 1. Metodología propuesta para la reconstrucción 3D y la comparativa entre la FTP y la WTP.

Para hacer la comparación de los métodos, la medición del error es implementada en el último paso, esta medición se llevará a cabo solo con el objeto virtual, es decir, al 
objeto virtual original se le restará el objeto obtenido de la reconstrucción para ambos métodos, obteniendo así el error haciendo así, la validación de los métodos aplicados.

La Fig. 1 describe el método propuesto para enmarcar los resultados del presente trabajo.

En la metodología hay un paso (4), que aplica Perfilometría Wavelet a una determinada imagen para su procesamiento, para llevar a cabo esta etapa es necesario formalizar la Transformada Wavelet, que para el caso del presente trabajo es la Transformada Morlet Compleja, debido a que ésta tiene una mejor respuesta comparativamente con respecto a otras transformadas Wavelets Complejas, definidas para el mismo fin López, 2012 [17]. Esta función está definida mediante

$$
\Psi(x)=\pi^{-1 / 4} \exp \left(i 2 \pi w_{0} x\right) \exp \left(-x^{2} / 2\right)
$$

donde:

$$
\operatorname{morl}(x)=e^{-x / 2} \cdot \cos (5 x),
$$

donde, $i=\sqrt{-1}$, y $w_{0}=$ Frecuencia de la Wavelet Morlet.

La importancia de esta transformada radica en que es una función continua y compleja, y esto permite hacer la descomposición de la señal (la imagen) en su parte real e imaginaria, de aquí se tiene un conocimiento mayor de la imagen. Esta función será la función madre que alimentará a la Transformada Wavelet, y llevar a cabo el proceso llamado Perfilometría Wavelet.

\section{Resultados experimentales}

Para la metodología propuesta fueron seleccionados dos objetos virtuales, los cuales se muestran en la Fig. 2.

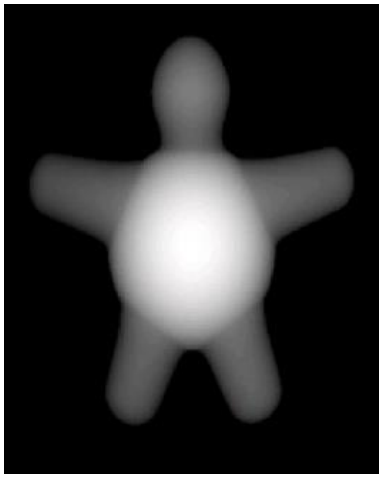

a)

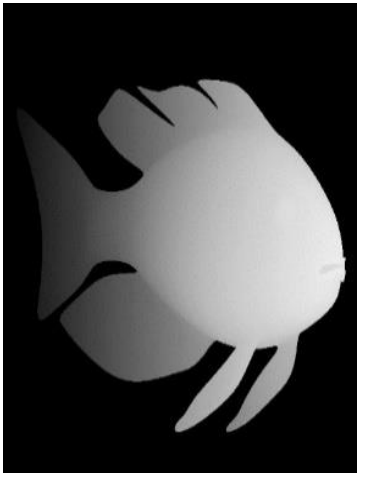

b)

Fig. 2. Objetos virtuales usados en este trabajo: a) Tortuga y b) Pez. 
Con la selección de los parámetros apropiados es factible obtener una buena reconstrucción $3 \mathrm{D}$ de objetos mediante el uso de algoritmos simples de desdoblamiento de fase, como se muestra en la Figura 3.

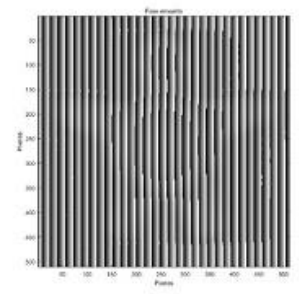

a)

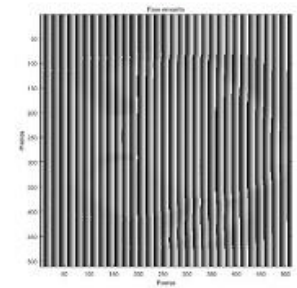

d)

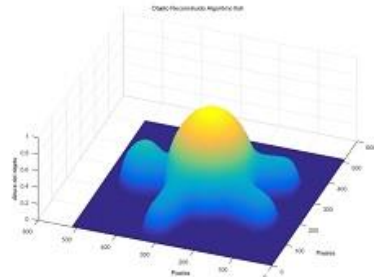

b)

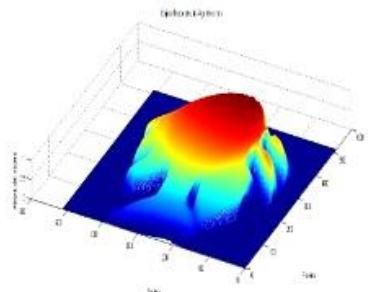

e)

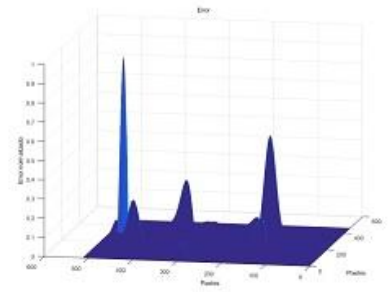

c)

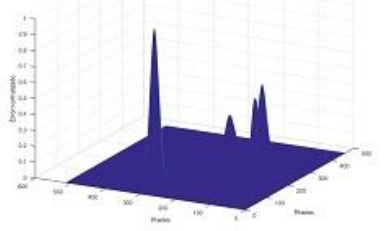

f)

Fig. 3. a) y d) Muestran la fase envuelta de los objetos, b) y e) muestran el objeto reconstruido y c) y f) muestran el error, el cual se obtiene restando el objeto original al objeto reconstruido.

Después de realizadas las pruebas con los objetos virtuales, el proceso es implementado a un objeto real, mostrado en la Fig. 4.

La Figura 5(a) muestra el mapa de fase obtenido, es el mapa de fase o fase envuelta la que contiene la información de la altura del objeto, para posteriormente obtener la fase desenvuelta, que es la reconstrucción 3D del objeto, el cual se muestra en 5 (b). Con objetos reales, el error no se maneja, dado que no se tiene la facilidad de hacer la diferencia entre el objeto original y el reconstruido.

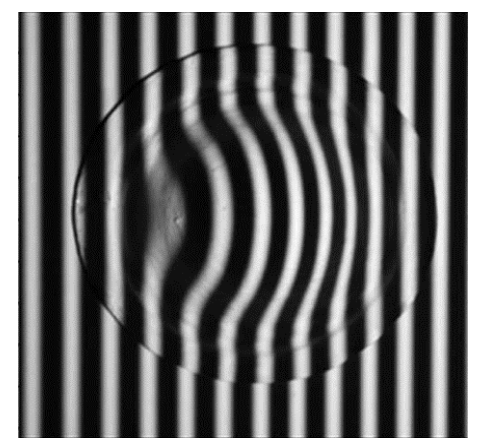

Fig. 4. Objeto Real con 12 franjas proyectadas.

La Fig. 5 muestra la reconstrucción 3D del objeto real utilizando la Wavelet Morlet. 


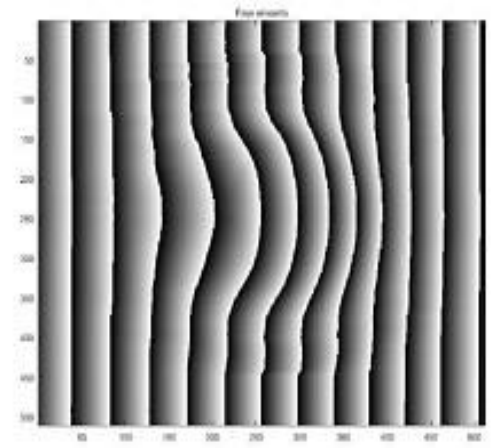

a)

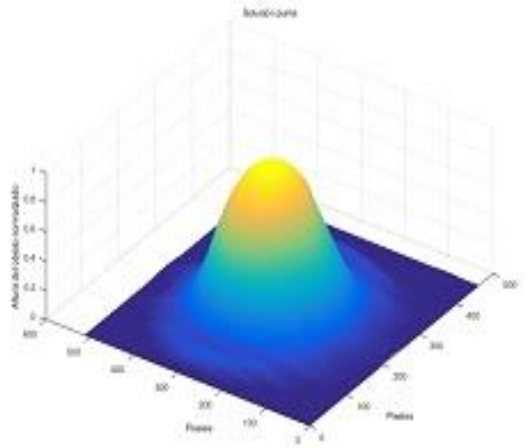

b)

Fig. 5. a) Fase envuelta y b) Reconstrucción 3D del objeto con wavelet Morlet.

En la Fig. 6 y 7 se muestran la reconstrucción de los objetos virtual y real respectivamente, pero utilizando la perfilometría de Fourier. En el caso de la Fig. 6, en la reconstrucción 3D de los objetos se presentaron ciertas ondulaciones, tanto en la base, así como en algunas partes de los objetos.

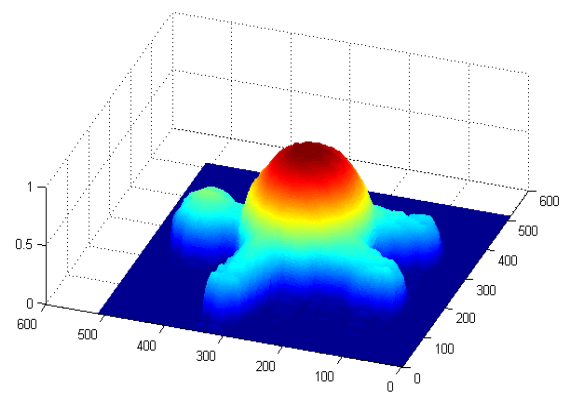

a)

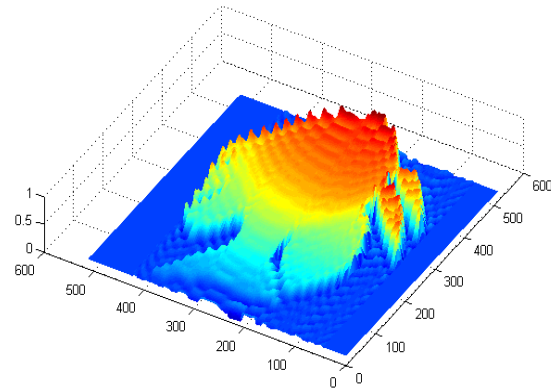

b)

Fig. 6. Reconstrucción 3D de objetos virtuales utilizando Perfilometría de Fourier: a) Tortuga, b) Pez.

Nótese como el mapa de fase, mostrado en la Fig. 7 a) tiene algunas zonas en donde se hace más gruesa una de las líneas proyectadas sobre el objeto, afectando este mapa de fase y posteriormente cuando se lleva a cabo la reconstrucción 3D del objeto completo, se obtiene una zona donde el objeto reconstruido tiene una pequeña deformación, la cual no existe en el objeto original. Esta deformación no está presente en la reconstrucción utilizando la Wavelet Morlet.

Adicionalmente, en la base del objeto se llegan a notar algunas ondulaciones en el objeto reconstruido utilizando la Perfilometría de Fourier como se observa en la Fig. 6 b), mientras que en la Fig. 5 b) la base del objeto reconstruido no tiene ondulaciones. 


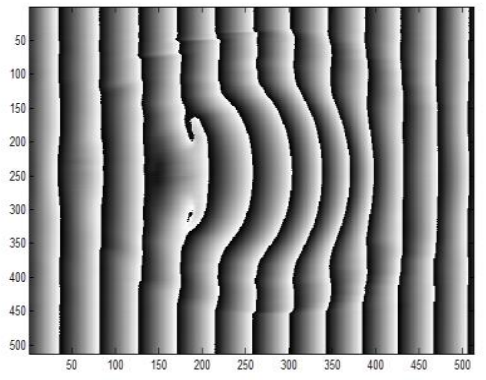

a)

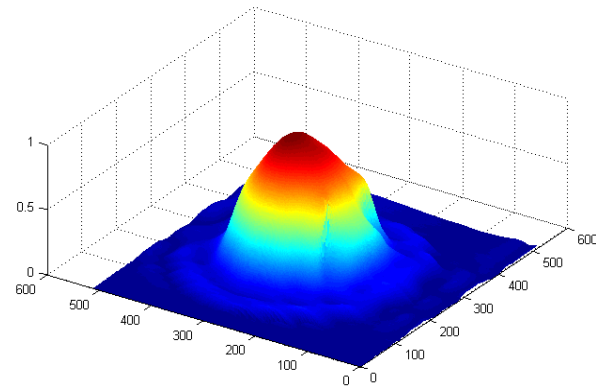

b)

Fig. 7. a) Fase envuelta y b) Reconstrucción 3D del objeto con Perfilometría de Fourier.

\section{Conclusiones y trabajo futuro}

En el presente artículo se propuso el uso de los métodos de Perfilometría de Fourier y Perfilometría Wavelet para la reconstrucción 3D de objetos. Se presenta una comparativa entre el desempeño de ambos métodos, implementando la selección de los mejores parámetros para la wavelet Morlet en la Perfilometría Wavelet, los cuales siendo seleccionados de forma adecuada, reducen el ruido en el mapa de fase de los objetos a reconstruir. Dentro de estos parámetros se considera el análisis de la estimación de fase mediante la frecuencia espacial $f_{0}$ determinada por el número de franjas proyectada sobre el objeto. Este análisis es utilizado para llevar a cabo el filtrado en esa frecuencia dentro de la Wavelet Morlet.

La metodología propuesta hace una distinción entre objetos reales y virtuales, así como la determinación de la frecuencia espacial $f_{0}$ si el caso de entrada es un objeto virtual. Los mejores resultados obtenidos de las pruebas llevadas a cabo en los objetos virtuales, fueron implementados en objetos reales, obteniendo los mejores resultados con la Perfilometría Wavelet. Con esto se puede apreciar que el uso de la Perfilometria Wavelet muestra un mejor desempeño que la Perfilometria de Fourier en este caso.

Como trabajo futuro se tiene la implementación y/o uso de algoritmos más robustos de desdoblamiento de fase, con el propósito de reducir el error en la reconstrucción 3D de objetos, independientemente del método a utilizar (Fourier o Wavelet).

\section{Referencias}

1. Asundi, A., Wensen, Z.: Fast phase-unwrapping algorithm based on a gray-scale mask and flood fill. Applied optics, Vol. 37, No. 23, pp. 5416-5420 (1998)

2. Mitsuo, T., Hideki, I., Seiji, K.: Fourier-Transform method of fringe pattern analysis for computed-based topography and interferometry. J.Opt. Soc.Am., Vol. 72, No. 1, pp. 156160 (1982)

3. $\mathrm{Wu}, \mathrm{L} .:$ Research and development of fringe projection-based methods in 3D shape reconstruction. Journal of Zhejiang University SCIENCE A, pp. 1026-1036 (2006) 
Propuesta de reconstrucción $3 d$ de objetos basado en la selección de los mejores parámetros ...

4. Pedraza, J. C., Rodriguez, W., Barriga, L.: Image Processing for 3D Reconstruction using a Modified Fourier Transform Profilometry Method. MICAI 2007, LNAI 4827, SpringerVerlag, Berlin Heidelberg, pp. 705-712 (2007)

5. Zhang, Z., Jing, Z., Wang, Z., Kuang, D.: Comparison of Fourier transform, windowed Fourier transform and wavelet transform methods for phase calculation at discontinuities in fringe profilometry. Optical and Lasers Engineering, Vol. 50, pp. 1152-1160 (2012)

6. Berryman, F., Pynsent, P., Cubillo, J.: A theoretical Comparison of three fringe analysis methods for determining the three-dimensional shape of an object in the presence of noise. Optics and Lasers in Engineering, Vol. 39, pp. 35-50 ( 2003)

7. Pramod, R.: Digital Speckle Pattern Interferometry and related Techniques. Edit. Wiley (2001)

8. Itoh, K.: Analysis of the phase unwrapping algorithm. Applied Optics, Vol. 21, No. 14, pp. 2470-2486 (1982)

9. Zhong, J., Weng, J.: Spatial carrier-fringe pattern analysis by means of wavelet transform: wavelet transform profilometry. Applied optics. Vol. 43, No. 26, pp. $4993-4998$ (2004)

10. Gdeisat, M. A., Abid, A., Burton, D. R., Lalor, M. J., Lilley, F., Moore, C., Qudeisat, M.: Spatial and temporal carrier fringe pattern demodulation using the one-dimensional continuous wavelet transform: recent progress, challenges, and suggested developments. Optics and Lasers in Engineering, Vol. 47, No. 12, pp. 1348-1361 (2009)

11. Xiang, Z., Hong, Z.: Three-Dimensional Shape Measurement Using Maar Wavelet Transform. In: Proceedings of ICIRA 2008, Part I LNAI 5314, pp. 753-760 (2008)

12. Qiang, Z., Wenjing, C., Yan, T.: Method of choosing the adaptive level of discrete wavelet decomposition to eliminate zero component. Optics Communication, pp. 778-785 (2008)

13. Abid, A. Z., Gdeisat, M. A., Burton, D. R., Lalor, M. J., Abdul-Rahman, H. S., Lilley, F.: Fringe pattern analysis using a one-dimensional modified Morlet continuous wavelet transform In Photonics Europe. International Society for Optics and Photonics, pp. 70000Q-70000Q (2008)

14. Fernandez, S., Gdeisat, M. A., Salvi, J., Burton, D. R.: Automatic window size selection in windowed Fourier transform for 3D reconstruction using adapted mother wavelets. Optics Communications, Vol. 284, No. 12, pp. 2797-2807 (2011)

15. Cortés, J. A., Garzón, H. B. C., Chaves, J. A.: Del Análisis de Fourier a las WaveletsTransformada Continua Wavelet (CWT). Scientia et Technica, Vol. 1, No. 37 (2007)

16. Arellano, P.: Missing information in remote sensing: wavelet approach to detect and remove clouds and their shadows. International Institute for Geo-Information Science and Earth Observation, Enschede, The Netherlands (2003)

17. López, T.: Estudio comparativo entre tipos de transformada wavelet para su uso en reconstrucción tridimensional. Doctoral dissertation (2012) 